\title{
Reconstitution and substrate specificity of the thioether-forming radical S-adenosylmethionine enzyme in freyrasin biosynthesis
}

\author{
Timothy W. Precord ${ }^{\dagger, \ddagger}$, Nilkamal Mahanta ${ }^{\dagger, \ddagger, \S}$, Douglas A. Mitchell ${ }^{\dagger, \ddagger},{ }^{\star}$ \\ tDepartment of Chemistry, University of Illinois at Urbana-Champaign, Urbana, Illinois 61801, \\ USA. \\ ‡Carl R. Woese Institute for Genomic Biology, University of Illinois at Urbana-Champaign, Urbana, \\ Illinois 61801, USA.
}

\begin{abstract}
The radical non-a-carbon thioether peptides (ranthipeptides) are a newly described class of ribosomally synthesized and post-translationally modified peptide (RiPP). Ranthipeptide biosynthetic gene clusters are characterized by a Cys-rich precursor peptide and a radical $S$ adenosylmethionine (rSAM)-dependent enzyme that forms a thioether linkage between a Cys donor and an acceptor residue. Unlike the sulfur-to- $a$-carbon linked thioether peptides (sactipeptides), known ranthipeptides contain thioethers to either the $\beta$ - or $\gamma$-carbon (i.e. non- $a$ carbon) of an acceptor residue. Recently, we reported the discovery of freyrasin, a ranthipeptide from Paenibacillus polymyxa, which contains six thioethers from Cys- $\mathrm{X}_{3}$-Asp motifs present in the precursor peptide (PapA). The linkages are exclusively to the $\beta$-carbon of Asp (S-C $\beta$ ). In this report, we performed mutational analysis of PapA and the cognate thioether-forming rSAM enzyme (PapB) to define the substrate scope. Using a mass spectrometry-based activity assay, our data show that PapB is intolerant towards Ala and Asn in the acceptor position but will tolerate Glu-containing variants. NMR spectroscopic data of a Glu variant demonstrated that the thioether linkage was to the 4-position of Glu ( $\mathrm{S}-\mathrm{C} \gamma)$. Furthermore, we demonstrate that PapB is intolerant to expansion and contraction of the thioether motifs $\left(\right.$ Cys- $\left.X_{n}-A s p, n=2,4\right)$, although a minimal substrate featuring only one Cys- $\mathrm{X}_{3}$-Asp motif was competent for thioether formation. Akin to the sactipeptides, PapB was dependent on a RiPP recognition element (RRE) to bind the cognate precursor peptide, with deletion resulting in loss-of-function in vivo. The activity of PapB could be restored in vivo by supplying the excised RRE in trans. Finally, we reconstituted the activity of PapB in vitro, which lead to modification of all six Cys residues in PapA. These studies provide insights into ranthipeptide biosynthesis and expand our understanding of rSAM enzyme chemistry in natural product biosynthesis.
\end{abstract}

\footnotetext{
*corresponding author Phone: 1-217-333-1345; Fax: 1-217-333-0508; douglasm@illinois.edu, Address: 600 South Mathews Avenue, Urbana, Illinois 61801, USA.

Present address: Department of Chemistry, Indian Institute of Technology, Dharwad, Karnataka, India, 580011 ASSOCIATED CONTENT

The Supporting Information is available free of charge on the ACS Publications website at DOI: 10.1021/acschembio.9b00457. and contains a description of experimental methods, and the supporting spectroscopic data collected for freyrasin variants.

The authors declare no competing financial interest.
} 


\section{Introduction}

Ribosomally synthesized and posttranslationally modified peptides (RiPPs) are a class of natural product with diverse structural features and biological activities, including antimicrobial, antitumor, immunosuppressive, and antinociceptive properties. ${ }^{1}$ RiPP biosynthesis follows a common logic wherein a ribosomal peptide undergoes modification by its associated biosynthetic enzymes, which are frequently encoded in a biosynthetic gene cluster (BGC). ${ }^{2}$ RiPP biosynthesis is typically guided by the N-terminal portion of the precursor peptide, termed the leader peptide, which facilitates the association of the precursor peptide and the modifying enzymes. ${ }^{3}$ To engage the leader peptide, the majority of prokaryotic RiPP biosynthetic pathways employ a $\sim 90$-residue domain referred to as the RiPP recognition element (RRE). ${ }^{4}$ In RRE-containing RiPP pathways, the RRE domain is frequently indispensable for substrate modification. The $\mathrm{C}$-terminal portion of the precursor peptide, termed the core peptide, receives the post-translational modifications. After the installation of leader peptide-dependent modifications, the leader region is removed and secondary tailoring of the natural product often occurs. ${ }^{5}$ A large variety of remarkable chemical modifications are observed in RiPPs and they are partitioned into classes based on these hallmark modifications. ${ }^{1,6}$

The ranthipeptides (radical non-a-carbon thioether peptides) are a recently reported class of RiPP characterized by the presence of a Cys-rich precursor peptide and a locally encoded radical $S$-adenosylmethionine (rSAM)-dependent enzyme (PF04055, Figure 1). ${ }^{7}$ Although they bear a superficial resemblance to the sactipeptides, which contain sulfur-to-a-carbon thioether linkages, the ranthipeptide-forming rSAM enzymes install a thioether modification at a non- $a$ carbon (i.e. the side chain) of an acceptor residue. ${ }^{7,8}$ Given their rSAMdependent formation, ranthipeptides are distinguished from the structurally similar lanthipeptides, which contain $\beta$-thioether linkages to dehydrated Ser/Thr residues via a Michael-like nucleophilic addition. ${ }^{9}$ rSAM enzymes, constituting one of the largest enzyme superfamilies, ${ }^{10}$ are present in a large number of RiPP biosynthetic pathways and carry out multiple difficult chemical transformations. ${ }^{11}$ They share the common usage of a [4Fe-4S] cluster to reductively cleave SAM to produce a highly reactive 5'-deoxyadenosyl (5'-DA) radical. ${ }^{10-13}$ The radical then typically abstracts a hydrogen atom from the substrate, priming it for the formation of the desired chemical modification. In addition to the [4Fe- $4 \mathrm{~S}]$ cluster associated with the rSAM domain, ranthipeptide synthases contain two additional [4Fe-4S] clusters within a C-terminal auxiliary domain. This domain is found in a variety of rSAM enzymes, including many involved in natural product biosynthesis, and has been dubbed a SPASM domain (for its presence in rSAM enzymes that modify subtilosin A, pyrroloquinoline quinone, anaerobic sulfatase, and mycofactocin, PF13186). ${ }^{14-18}$ Structural analysis of thermocellin, a ranthipeptide from Hungateiclostridium thermocellum, in contact with its cognate rSAM ( $\mathrm{CteB})$ indicates that a potential donor Cys residue is ligated to the first auxiliary [4Fe-4S] cluster. ${ }^{18}$ This result has previously been reported for other classes of SPASM domain-containing rSAM enzymes and suggests that at least one auxiliary cluster may play a role in substrate coordination for proper thioether formation. ${ }^{15}$ Other lines of evidence support a role for the auxiliary Fe-S clusters in electron transfer from the substrate. Here, we tentatively consider the first auxiliary $[4 \mathrm{Fe}-4 \mathrm{~S}]$ cluster to be acting within a 
coordinating role rationalized by the high sequence similarity of CteB with the other ranthipeptides. Future research on ranthipeptide biosynthesis may support or refute this mechanism.

Our group has recently employed Rapid ORF Description \& Evaluation Online (RODEO) ${ }^{19}$ to conduct a bioinformatic survey on the sactipeptide and ranthipeptide classes. Sequence analysis of the rSAM enzymes revealed that many ranthipeptide synthases bear high sequence similarity to quinohemoprotein amine dehydrogenase maturase (QhpD). ${ }^{7}$ The QhpD-like subset of ranthipeptides was previously reported in a computational study ${ }^{20}$ and the peptides were initially termed SCIFFs (six Cys in forty-five residues) based on a conserved precursor peptide sequence. $\mathrm{QhpD}$ is an rSAM enzyme necessary for the maturation of quinohemoprotein amine dehydrogenase (QHNDH), a protein complex found in some Gram-negative bacteria (Figure S2). ${ }^{21-24} \mathrm{QhpD}$ catalyzes the formation of three intramolecular thioether linkages in the small subunit of QHNDH (QhpC). ${ }^{25}$ Importantly, these thioether linkages are installed on the side chains of three acidic residues in QhpC, with two linkages between Cys and Asp at the $\beta$-carbon and one linkage to the $\gamma$-carbon of Glu. ${ }^{22-24}$ Indeed, the high level of sequence similarity between the "SCIFF" rSAM enzymes and QhpD was an early indicator that SCIFFs might not be sactipeptides, but instead belonged to a new class of RiPP and have been recategorized as members of the ranthipeptide class. ${ }^{7}$ Thus far, there has been a single report of a non-SCIFF ranthipeptide whose maturase, NxxcB, catalyzes thioether formation between the Cys thiol and the $\beta$ carbon of an Asn three residues upstream. ${ }^{26}$ BLAST-based sequence comparison between QhpD from Paracoccus denitrificans (SDJ52620.1) and NxxcB (WP_018375754.1) yields a query coverage of $42 \%$ with $45 \%$ sequence similarity over the aligned region (bit score 52 ). Bioinformatics analysis predicts additional non-SCIFF ranthipeptides remain to be discovered.

Here, we further characterize freyrasin, a ranthipeptide from Paenibacillus polymyxa ATCC $842 .{ }^{7}$ Like the previously reported thermocellin and Tte $1186 \mathrm{a},{ }^{18,27}$ the freyrasin rSAM maturase, PapB (WP_019688962.1), has considerably higher sequence similarity to QhpD than does NxxcB (BLAST query coverage of $94 \%$ with $50 \%$ sequence similarity, bit score 229). The freyrasin precursor (PapA) contains six Cys- $X_{3}$-Asp motifs where $X$ is any amino acid residue. PapB cyclizes these motifs by forming a thioether linkage between the Cys thiol and the Asp acceptor at the $\beta$-carbon, reminiscent of QhpD (Figure 1). The novel RiPP thioether linkage was verified by a combination of bioinformatic analysis, high-resolution and tandem MS, and multidimensional NMR. ${ }^{7}$ Given the recent structural revision and bioinformatics survey, we sought to determine the substrate tolerance of a QhpD-like ranthipeptide rSAM enzyme. Here, we analyzed the substrate selectivity of PapB through extensive alteration of the PapA precursor peptide and co-expression with PapB in E. coli. We further characterized an Asp to Glu variant of PapA that was successfully crosslinked by PapB and found that the Cys thiol was crosslinked to the $\gamma$-carbon of Glu, mirroring the activity of QhpD in installing $\beta$ - and $\gamma$-carbon linkages to Asp and Glu, respectively. Additionally, we reconstituted the activity of PapB in vitro and found that the reaction was dependent on SAM and an external reductant. Finally, we demonstrate that PapA modification was dependent on the presence of an RRE in PapB that facilitated the association of PapB with the PapA precursor. 


\section{Results and Discussion}

\section{Substrate tolerance of the PapB ranthipeptide synthase}

To evaluate the substrate tolerance of PapB, positions of PapA were varied and co-expressed in E. coli with PapB (Figure 2). First, six individual replacements of Asp to Ala of PapA were prepared as fusions to maltose-binding protein (MBP). After induction of protein expression, amylose affinity chromatography was used to purify the MBP-tagged PapA variants prior to trypsinolysis and analysis by matrix-assisted laser desorption/ionizationtime-of-flight mass spectrometry (MALDI-TOF-MS). In each case, a mass loss of $10 \mathrm{Da}$ was observed, compared to the $-12 \mathrm{Da}$ observed in modification of the wild-type (WT), indicating successful installation of 5 thioether linkages. Furthermore, the resulting peptide core regions were singly alkylated upon iodoacetamide (IAA) treatment, suggesting the presence of a single free thiol in each case (Figure S3). High-resolution and tandem MS of each Asp to Ala variant confirmed that the remaining Cys- $\mathrm{X}_{3}$-Asp motifs were processed by PapB as they were in the WT co-expression experiment (Figures S9-S14). Additionally, variation of the sixth ring Asp (D30A) resulted in a subpopulation of product displaying a mass loss of $8 \mathrm{Da}$. This species was doubly alkylated by iodoacetamide (IAA) and was analyzed by tandem MS (Figure S15). The second alkylation event occurred at the fourth ring Cys17, suggesting that this position has lower priority during heterologous expression in E. coli.

We were interested in determining the importance of the carboxylate group in the PapA acceptor residue of each Cys- $\mathrm{X}_{3}$-Asp motif for PapB processing. Therefore, all individual Asp to Asn variants were prepared and co-expressed with PapB as above. After purification and trypsin digestion, the same result was observed as for the Asp to Ala scan for every position. Each core peptide had a mass loss of $10 \mathrm{Da}$ and was alkylated once by IAA (Figures 2 and S4). As with the D30A variant, processing of the D30N variant yielded a doubly alkylated subpopulation after IAA labeling, presumably for the same reason. These data suggest that the carboxylate moiety in the acceptor residue may be indispensable for thioether crosslink formation by PapB.

Based on our understanding of QhpD activity, we predicted that PapB would catalyze the formation of a thioether crosslink between Cys and Glu. Each acceptor Asp of PapA was individually substituted with Glu and co-expressed with PapB. The resultant core peptides underwent processing by PapB with a mass loss of $12 \mathrm{Da}$, consistent with the formation of six thioether linkages, and the resulting products were unreactive towards IAA (Figures 2 and S5). Variant D6E was further analyzed by high-resolution and tandem MS, which confirmed an identical thioether linkage topology as WT freyrasin (Figure S20). These data support a substrate recognition mechanism where PapB uses the carboxylate moiety on the acceptor residue to properly coordinate the $\mathrm{Cys}-\mathrm{X}_{3}$-Asp motif for thioether formation.

To evaluate whether PapB activity was disturbed by the removal of donor Cys residues, each Cys to Ala variant was prepared and the resultant peptides were co-expressed with PapB. After purification and trypsin digestion, MALDI-TOF-MS analysis showed that each peptide had a mass loss of $10 \mathrm{Da}$ compared to the unmodified sequence, indicating the formation of five thioether rings (Figure 2). All were unreactive towards labeling by IAA due to the lack 
of a free thiol and four produced sufficient fragmentation for analysis by tandem MS (Figure S6). Those four (C2A, C11A, C17A, and C20A) were all modified by PapB at the remaining Cys- $\mathrm{X}_{3}$-Asp motifs in a manner identical to WT freyrasin (Figures S16-S19).

These data suggest that PapB processes each Cys- $\mathrm{X}_{3}$-Asp motif independently and skips over any ring variant that does not contain the proper coordinating residues. We next probed if PapB would tolerate $\mathrm{a}+1$ and -1 ring expansion/contraction. PapA-Ala4 was removed for the contracted ring variant to yield a Cys- $\mathrm{X}_{2}$-Asp motif (CSND), which was then coexpressed with PapB. The resultant peptide had a mass loss of 12 Da but displayed a broader isotopic distribution pattern by MALDI-TOF-MS, suggesting a -10 Da species was present (Figure S8). After reaction with IAA, a significant amount of alkylated product was observed, indicating that PapB inefficiently processed the ring contraction variant. The fully modified product was analyzed by tandem MS, which suggested all ring topologies, including the contracted ring, had been installed by PapB in a WT-like manner (Figure S26). In the singly alkylated product, it seemed likely that the contracted ring was the site of labeling by IAA, but this was not confirmed experimentally as the peptide did not produce sufficiently intense fragment ions for tandem MS analysis. Next, an additional Ala at position 3 was introduced to produce an expanded Cys- $\mathrm{X}_{4}$-Asp motif (CSAAND). After coexpression, the peptide exhibited a mass loss of $10 \mathrm{Da}$ and was monoalkylated by IAA, indicating that PapB was intolerant to this ring expansion. Tandem MS analysis confirmed that the location of the other five rings was preserved (Figure S25). These data further support that a three-residue loop size is required for PapB to effectively catalyze thioether formation. While PapB likely has a preferred order of ring installation, in vivo catalysis of any Cys- $\mathrm{X}_{3}$-Asp motif was not predicated on the successful installation of any other thioether linkage in the core.

Finally, to determine the minimal substrate for PapB, we introduced a stop codon after the first Cys- $\mathrm{X}_{3}$-Asp motif in PapA. After co-expression with PapB, a mass loss of 2 Da was observed in the peptide which was also devoid of labeling by IAA (Figure S7). These results demonstrate that PapB efficiently installs a thioether linkage in a substrate that contains only the leader peptide and one Cys- $\mathrm{X}_{3}$-Asp motif.

\section{Freyrasin D6E variant is a $\mathrm{S}-\mathrm{C} \boldsymbol{\gamma}$ crosslinked peptide}

Given that QhpD catalyzes the formation of a Cys-Glu (S-C $\gamma$ ) thioether linkage in QhpC, ${ }^{25}$ we hypothesized that PapB might also be capable of forming such a linkage (Figure 3 and Figure S2). To evaluate this, we prepared PapA-D6E and, after a larger scale co-expression with PapB, isolated sufficient quantities to enable multidimensional NMR analysis (Supplemental Methods). The previously reported ${ }^{1} \mathrm{H}-{ }^{1} \mathrm{H}$ Total Correlation Spectroscopy (TOCSY) for freyrasin ${ }^{7}$ was used alongside the ${ }^{1} \mathrm{H}_{-}{ }^{1} \mathrm{H}$ TOCSY for the D6E variant to assign residues based on the amide region cross-peaks in the D6E spectrum (Figure S27). A ${ }^{1} \mathrm{H}-{ }^{1} \mathrm{H}$ Nuclear Overhauser Effect Spectroscopy (NOESY) spectrum of D6E freyrasin was used to confirm the sequence of residues in the peptide core (Figure S28). Through-space amideamide correlations were observed for all residues with the exception of Cys8 and Tyr9. In cases where high amino acid multiplicity resulted in amide spin system overlap (Table S1), residues could be assigned using amide-amide through-space correlations. The residue of 
interest, Glu6, was assigned with high confidence in the ${ }^{1} \mathrm{H}^{1}{ }^{1} \mathrm{H}$ TOCSY as there are no other Glu or Gln residues in the molecule (Figure 3). Presence of side chain signals in the amide region in the ${ }^{1} \mathrm{H}-{ }^{1} \mathrm{H}$ TOCSY supports a non-a-carbon thioether linkage and the chemical shift values in the Glu spin system were consistent with a linkage at $\mathrm{C} 4$ of $\mathrm{Glu}(\mathrm{C} \gamma)$. The signal at $3.64 \mathrm{ppm}$ was downshifted by proximity to the crosslinked sulfur atom while the methylene signals at 1.78 and $2.11 \mathrm{ppm}$ were consistent with the shift values of the $\beta$ hydrogens in free Glu. This conclusion is further supported by NOE contacts between Cys 2 and Glu6 in the ${ }^{1} \mathrm{H}-{ }^{1} \mathrm{H}$ NOESY spectrum wherein both Cys $\beta$-hydrogens exhibit interactions with the Glu $\gamma$-hydrogen. Additionally, the Glu hydrogen atom at the crosslink position has a strong NOE correlation to the methylene hydrogens on Glu6 but a weak NOE to the ahydrogen, supporting its identity as the $\gamma$-hydrogen. These data are complicated by splitting in the $\gamma$ and $\beta$ spin systems but are consistent with the installation of a S-C $\gamma$ linkage in the variant $\mathrm{Cys}-\mathrm{X}_{3}$-Glu motif.

\section{Modification of the freyrasin precursor is RRE-dependent}

As many RiPP classes are dependent on a physical interaction between the precursor leader peptide and an RRE domain, ${ }^{4}$ we reasoned that freyrasin may similarly show RREdependence for modification by PapB. Domain analysis of PapB indicated the presence of an N-terminal helix-turn-helix domain with sequence similarity to PqqD (PF05402), a discrete winged helix-turn-helix protein necessary for the maturation of pyrroloquinoline quinone cofactor (Figure S30). ${ }^{4,28,29}$ Sequence similarity to PqqD is frequently used as a bioinformatic identifier of RRE domains. ${ }^{4}$ In PapB, this domain encompasses the first 86 residues of PapB and ends shortly before the beginning of the rSAM domain. Ablation of this domain from PapB (PapB- $\Delta 1-85)$ resulted in a protein incapable of thioether formation during co-expression in E. coli (Figure S30). When the excised PapB RRE domain (residues 1-85) was co-expressed with PapA and PapB- $\Delta 1-85$, the substrate displayed a mass loss of $12 \mathrm{Da}$ and gave a fragmentation pattern identical to WT freyrasin (Figure S24). As addition of the RRE in trans was sufficient to rescue the activity of PapB, the RRE was necessary to promote association with PapA.

We next sought to determine which residues of the leader peptide comprise the recognition sequence (i.e. the residues directly in contact with the RRE). Initially, we attempted to probe the leader peptide-RRE interaction using a fluorescence polarization (FP) method reported previously. ${ }^{4,30-32}$ Unfortunately, all attempts using FP were unsuccessful using either the excised RRE domain or the full length PapB protein (data not shown). We next attempted to determine binding residues through saturation transfer difference NMR (STD-NMR). These experiments also did not yield data indicative of binding, suggesting an incompatibility of PapB with these in vitro techniques. As an alternative to FP and STD-NMR, we assessed the relative importance of leader peptide residues by mutational analysis and extent of processing during E. coli expression. The recognized portion of the leader peptide of CteA (thermocellin precursor, ABN52141.1) responsible for binding the $\beta 3$ strand in the $\mathrm{CteB}$ RRE has been reported (Figure S29). ${ }^{18}$ Furthermore, sequence alignment of ranthipeptides that contain Cys- $\mathrm{X}_{3}$-Asp motifs indicate that Asn(-13) was perfectly conserved in those precursors, suggesting it might play a binding role (Figure 4). Therefore, an Ala scan of the leader peptide was performed from residues Leu(-17) to Ile(-11), a region which aligns 
with the $\beta 3$ strand-binding region in CteA. After co-expression of the Ala variants with PapB, the resultant core peptides were analyzed by MALDI-TOF-MS. As a negative control, we prepared a construct in which all residues in the leader peptide were removed (Figure S31). This leader-deletion construct was not accepted as a substrate by PapB. All Alasubstituted leader peptide variants were processed in a fashion identical to WT (Figure 4) with the exception of $\mathrm{N}(-13) \mathrm{A}$, which had a broadened isotopic pattern indicating a mixture of products had formed. Upon IAA treatment, 0-6 alkylation events were observed on PapA-N(-13)A. Subjecting the products with 0-2 alkylation events to tandem MS analysis yielded ions of sufficient intensity to localize the incompletely processed positions. The 0 alkylation product ( $-12 \mathrm{Da}$ species) was of low intensity compared to WT and contained thioether linkages in the correct locations (Figure S21). The singly alkylated product was missing a thioether linkage at Cys17, indicating that this position is likely the last thioether linkage formed by PapB in vivo, consistent with the previously mentioned observations with the D30A variant (Figures S14 and S22). The doubly alkylated species displayed an IAA adduct at Cys17 with the second labeling event occurring primarily at Cys11 with minor labeling at Cys26 also observed (Figure S23). These data suggest that ring 4 was processed last by PapB and that rings 3 and 6 were penultimately installed with greater leniency towards installation order.

\section{In vitro reconstitution of PapB activity}

To reconstitute freyrasin biosynthesis in vitro, we co-expressed MBP-tagged PapB with pSUF (plasmid containing the Fe-S cluster biosynthetic operon, suf) ${ }^{33}$ in E. coli and purified MBP-PapB under anaerobic conditions (Figure S1 and Supplemental Methods). Using the ferrozine ${ }^{34}$ and methylene blue assays, ${ }^{35}$ we found that purified PapB contained $9.8 \pm 0.2 \mathrm{Fe}$ and $10.5 \pm 0.4$ sulfides per monomer, suggesting that the enzyme harbors more than two [4Fe-4S] clusters. These data were consistent with the presence of a two [4Fe-4S] cluster SPASM domain in PapB. Further support for the presence of a SPASM domain in PapB is provided by a sequence alignment to structurally-characterized rSAM enzymes containing the SPASM auxiliary domain (Figure S37). To reconstitute the reaction, we treated PapA with PapB in the presence of SAM, dithionite, and dithiothreitol (DTT) under anaerobic conditions. The reaction mixture was analyzed by MALDI-TOF-MS and a product was observed at $\mathrm{m} / \mathrm{z} 3564 \mathrm{Da}$, consistent with the formation of six thioether bonds and our previous report. ${ }^{7}$ Omission of PapB, SAM, or dithionite from the reaction did not result in product formation. The biosynthetic reconstitution of freyrasin was also successful when using a biological reductant system comprised of flavodoxin, flavodoxin reductase, and NADPH instead of dithionite (Figure 5).

Reported rSAM enzymes involved in thioether formation use one [4Fe-4S] cluster to reductively cleave SAM to generate a reactive 5 '-DA radical. ${ }^{10}$ This radical then abstracts a hydrogen atom from the a-carbon of the acceptor residue of the precursor in sactipeptides (or, presumably, a non-a-carbon residue in ranthipeptides) to generate 5'-DA as the byproduct. An auxiliary [4Fe-4S] cluster in the SPASM domain has been postulated to oxidize the donor Cys sulfhydryl group to the thiyl radical, which then would recombine with the C-centered radical to form the class-defining thioether bond. ${ }^{14}$ However, the order of oxidation and recombination events in this mechanism remain speculative (Figure S35). 
To assess if PapB produced 5'-DA as a byproduct, we removed the proteins from the reaction mixture by precipitation/ultracentrifugation and subjected the supernatant to highperformance liquid chromatography (HPLC) analysis. 5'-DA was not detected in any of the control reactions individually lacking PapB, SAM, or dithionite. In the full reaction, the presence of 5'-DA was confirmed by co-elution with an authentic standard and electrospray ionization-liquid chromatography MS analysis (Figure S33). This suggests that the radicalmediated thioether bond formation in ranthipeptides is initiated akin to the mechanism operating in sactipeptide biosynthesis. ${ }^{18}$ Moreover, as a C-radical adjacent to a carbonyl group would stabilized by delocalization, the site of radical generation in the ranthipeptide could be rationalized as $\beta$-carbon in Asp and $\gamma$-carbon in Glu from this mechanistic hypothesis (Figure S35). However, the importance of the carboxylate moiety could also be defined as a coordinating site as discussed below.

To confirm the identity of the Cys residues involved in coordinating the rSAM [4Fe-4S] cluster during catalysis, we prepared PapB Cys to Ala variants and assessed the effect of these alterations on enzyme activity. The Cys residues in the canonical rSAM clusterbinding motif $\left(\mathrm{CX}_{3} \mathrm{CX}_{2} \mathrm{C}\right)$ were individually substituted with Ala (C119A, C123A and C126A). These PapB variants were expressed, purified anaerobically, and subjected to the above-described activity assays. As expected, none of these variants were active, suggesting the critical importance of these Cys residues in binding the essential [4Fe-4S] cluster (Figure S34).

\section{Analysis of Conserved Active Site Residue Arg372}

We next investigated whether we could determine residue(s) of PapB that were responsible for coordinating the acceptor Asp residue during the thioether cyclization reaction. The structure of CteB (thermocellin maturase, PDB code 5WGG) has been reported, ${ }^{18}$ which shows Gln364 within the active site that is close enough to be a plausible acceptorcoordinating residue (Thr in the case of CteA). Furthermore, upon analysis of our ranthipeptide database, ${ }^{7}$ we found that $\mathrm{Gln} 364$ is conserved in $99.5 \%$ of the surveyed thermocellin-like maturases (when conflating sequences $>90 \%$ identical at the amino acid level; 2,101 proteins represented by 827 sequences, Figure S36). This can be contrasted with the relatively high sequence variation in the surrounding residues. In freyrasin-like ranthisynthases, this residue is invariantly Arg amongst the 17 Cys- $\mathrm{X}_{3}$-Asp-modifying rSAMs we have previously cataloged. QhpD possesses a conserved Arg373 in the same region that upon replacement with Ala resulted in the reductive cleavage of SAM but no observable thioether linkage. ${ }^{25}$

Given these observations and the proximity of PapB-Arg372 to the auxiliary Fe-S clusterbinding Cys residues found in the SPASM domain, we reasoned that PapB-Arg372 would be the most probable residue for coordinating acceptor Asp residues of PapA. Therefore, we produced variants of PapB that contained substitutions at Arg372. When co-expressed with PapB-R372A, the purified peptide lacked modification, as assessed by MALDI-TOF-MS (Figure S36). When co-expressed with PapB R372Q (mirroring the Thr-coordinating residue of $\mathrm{CteB}$ ), fully modified PapA was detected, although with lower ion intensity relative to unmodified PapA. Taken together, these data suggest that Arg372 may coordinate the 
carboxylate moieties on the acceptor residues of PapA and QhpC. We predict that a similar phenomenon might govern the residue-specific modification of QhpC by QhpD.

Furthermore, we anticipate that CteB-Gln364 similarly acts to coordinate the acceptor Thr residue in CteA. Further structural and enzymatic characterization is necessary in order to fully establish the role of these residues in rSAM-catalyzed thioether formation.

\title{
Conclusion
}

In this work, we have presented the first characterization of the substrate scope of a SCIFFtype ranthipeptide maturase, PapB. Through the use of an in vivo expression system, we have demonstrated that PapB activity is leader peptide-dependent and requires a 3-amino acid spacer between the acceptor and donor residues for efficient thioether formation. While in vitro experimentation failed to yield conclusive results about the nature of the PapA leader peptide interaction with $\mathrm{PapB}$, in vivo experimentation confirmed engagement between the PapA leader and an N-terminal RRE domain on PapB. While PapB exhibits a preferred order of ring formation, these modifications can occur independently during expression in $E$. coli. As such, these ranthipeptide maturases could be attractive candidates for generating hybrid RiPPs that include Cys- $\mathrm{X}_{3}$-Asp/Glu thioether macrocycles. Our lab has previously leveraged a chimeric leader peptide design to bridge different RiPP classes in order to generate new-to-nature hybrid RiPP molecules with diverse structures. ${ }^{36}$ As PapB shows no obvious dependence on the identity of the three intervening residues, nor interdependence on ring installation order, it should be amenable for hybrid RiPP pathway engineering and library generation. However, it has been observed that an optimal spacing of three residues is required between the donor and acceptor residues in freyrasin for efficient thioether formation. This may be due to distance constraints between the SAM-cleaving [4Fe-4S] cluster and the Cys-coordinating auxiliary [4Fe-4S] cluster in the SPASM domain (if the auxiliary cluster does indeed act as a coordinating site in this way). Additionally, we have reconstituted the activity of PapB in vitro and shown it is active under anaerobic conditions. Finally, we probed the activity of PapB and confirmed its dependence on the rSAM [4Fe-4S]-ligating Cys residues and determined that Arg372 is critical for efficient substrate modification. Additional biochemical and structural studies are underway to gain further insight into substrate recognition and the precise ranthisynthase mechanism.

\section{Supplementary Material}

Refer to Web version on PubMed Central for supplementary material.

\section{ACKNOWLEDGEMENT}

\author{
We are grateful to L. Zhu (Univ. of Illinois) for assistance with NMR data acquisition. We further thank G. Hudson \\ and X. Guo (Univ. of Illinois) for acquisition of all tandem MS data. G. Hudson additionally provided revision and \\ editing suggestions for the manuscript. \\ Funding Sources \\ This work was supported by the David and Lucile Packard Fellowship for Science and Engineering (to D.A.M.) and \\ the Chemistry-Biology Interface Research Training Program (Grant T32 GM070421 to T.W.P.). Funds to purchase \\ the Bruker UltrafleXtreme MALDI TOF/TOF mass spectrometer were from the National Institutes of Health (S10 \\ RR027109 A).
}




\section{References}

(1). Arnison PG; Bibb MJ; Bierbaum G; Bowers AA; Bugni TS; Bulaj G; Camarero JA; Campopiano DJ; Challis GL; Clardy J; Cotter PD; Craik DJ; Dawson M; Dittmann E; Donadio S; Dorrestein PC; Entian KD; Fischbach MA; Garavelli JS; Göransson U; Gruber CW; Haft DH; Hemscheidt TK; Hertweck C; Hill C; Horswill AR; Jaspars M; Kelly WL; Klinman JP; Kuipers OP; Link AJ; Liu W; Marahiel MA; Mitchell DA; Moll GN; Moore BS; Müller R; Nair SK; Nes IF; Norris GE; Olivera BM; Onaka H; Patchett ML; Piel J; Reaney MJ; Rebuffat S; Ross RP; Sahl HG; Schmidt EW; Selsted ME; Severinov K; Shen B; Sivonen K; Smith L; Stein T; Süssmuth RD; Tagg JR; Tang GL; Truman AW; Vederas JC; Walsh CT; Walton JD; Wenzel SC; Willey JM; van der Donk WA Ribosomally Synthesized and Post-Translationally Modified Peptide Natural Products: Overview and Recommendations for a Universal Nomenclature. Nat. Prod. Rep 2013, 30, 108-160. [PubMed: 23165928]

(2). Ortega MA; van der Donk WA New Insights into the Biosynthetic Logic of Ribosomally Synthesized and Post-Translationally Modified Peptide Natural Products. Cell Chem. Biol 2016, 23, 31-44. [PubMed: 26933734]

(3). Oman TJ; van der Donk WA Follow the Leader: The Use of Leader Peptides to Guide Natural Product Biosynthesis. Nat. Chem. Biol 2010, 6, 9-18. [PubMed: 20016494]

(4). Burkhart BJ; Hudson GA; Dunbar KL; Mitchell DA A Prevalent Peptide-Binding Domain Guides Ribosomal Natural Product Biosynthesis. Nat. Chem. Biol 2015, 11, 564-570. [PubMed: 26167873]

(5). Funk MA; van der Donk WA Ribosomal Natural Products, Tailored To Fit. Acc. Chem. Res 2017, 50, 1577-1586. [PubMed: 28682627]

(6). Hudson GA; Mitchell DA RiPP Antibiotics: Biosynthesis and Engineering Potential. Curr. Opin. Microbiol 2018, 45, 61-69. [PubMed: 29533845]

(7). Hudson GA; Burkhart BJ; DiCaprio AJ; Schwalen CJ; Kille B; Pogorelov TV; Mitchell DA Bioinformatic Mapping of Radical S-Adenosylmethionine-Dependent Ribosomally Synthesized and Post-Translationally Modified Peptides Identifies New Ca, $\mathrm{C} \beta$, and $\mathrm{C} \gamma$-Linked ThioetherContaining Peptides. J. Am. Chem. Soc 2019, 141, 8223-8238.

(8). Fluhe L; Marahiel MA Radical S-Adenosylmethionine Enzyme Catalyzed Thioether Bond Formation in Sactipeptide Biosynthesis. Curr. Opin. Chem. Biol 2013, 17, 605-612. [PubMed: 23891473]

(9). Repka LM; Chekan JR; Nair SK; van der Donk WA Mechanistic Understanding of Lanthipeptide Biosynthetic Enzymes. Chem. Rev. 2017, 117, 5457-5520. [PubMed: 28135077]

(10). Broderick JB; Duffus BR; Duschene KS; Shepard EM Radical S-Adenosylmethionine Enzymes. Chem. Rev 2014, 114, 4229-4317. [PubMed: 24476342]

(11). Mahanta N; Hudson GA; Mitchell DA Radical S-Adenosylmethionine Enzymes Involved in RiPP Biosynthesis. Biochemistry 2017, 56, 5229-5244. [PubMed: 28895719]

(12). Bauerle MR; Schwalm EL; Booker SJ Mechanistic Diversity of Radical S-Adenosylmethionine (SAM)-Dependent Methylation. J. Biol. Chem 2015, 290, 3995-4002. [PubMed: 25477520]

(13). Mahanta N; Zhang Z; Hudson GA; van der Donk WA; Mitchell DA Reconstitution and Substrate Specificity of the Radical S-Adenosyl-Methionine Thiazole C-Methyltransferase in Thiomuracin Biosynthesis. J. Am. Chem. Soc 2017, 139, 4310-4313. [PubMed: 28301141]

(14). Benjdia A; Guillot A; Lefranc B; Vaudry H; Leprince J; Berteau O Thioether Bond Formation by SPASM Domain Radical SAM Enzymes: Ca H-Atom Abstraction in Subtilosin A Biosynthesis. Chem. Commun 2016, 52, 6249-6252.

(15). Grell TAJ; Goldman PJ; Drennan CL SPASM and Twitch Domains in S-Adenosylmethionine (SAM) Radical Enzymes. J. Biol. Chem 2015, 290, 3964-3971. [PubMed: 25477505]

(16). Barr I; Stich TA; Gizzi AS; Grove TL; Bonanno JB; Latham JA; Chung T; Wilmot CM; Britt RD; Almo SC; Klinman JP X-Ray and EPR Characterization of the Auxiliary Fe-S Clusters in the Radical SAM Enzyme PqqE. Biochemistry 2018, 57, 1306-1315. [PubMed: 29405700]

(17). Grell TAJ; Kincannon WM; Bruender NA; Blaesi EJ; Krebs C; Bandarian V; Drennan CL Structural and Spectroscopic Analyses of the Sporulation Killing Factor Biosynthetic Enzyme 
SkfB, a Bacterial AdoMet Radical Sactisynthase. J. Biol. Chem 2018, 293, 17349-17361. [PubMed: 30217813]

(18). Grove TL; Himes PM; Hwang S; Yumerefendi H; Bonanno JB; Kuhlman B; Almo SC; Bowers AA Structural Insights into Thioether Bond Formation in the Biosynthesis of Sactipeptides. J. Am. Chem. Soc 2017, 139, 11734-11744. [PubMed: 28704043]

(19). Tietz JI; Schwalen CJ; Patel PS; Maxson T; Blair PM; Tai H-C; Zakai UI; Mitchell DA A New Genome-Mining Tool Redefines the Lasso Peptide Biosynthetic Landscape. Nat. Chem. Biol 2017, 13, 470-478. [PubMed: 28244986]

(20). Haft DH; Basu MK Biological Systems Discovery in Silico: Radical S-Adenosylmethionine Protein Families and Their Target Peptides for Posttranslational Modification. J. Bacteriol 2011, 193, 2745-2755. [PubMed: 21478363]

(21). Adachi O; Kubota T; Hacisalihoglu A; Toyama H; Shinagawa E; Duine JA; Matsushita K Characterization of Quinohemoprotein Amine Dehydrogenase from Pseudomonas Putida. Biosci Biotechnol. Biochem 1998, 62, 469-478. [PubMed: 27315927]

(22). Datta S; Mori Y; Takagi K; Kawaguchi K; Chen Z-W; Okajima T; Kuroda S; Ikeda T; Kano K; Tanizawa K; Mathews FS Structure of a Quinohemoprotein Amine Dehydrogenase with an Uncommon Redox Cofactor and Highly Unusual Crosslinking. Proc. Natl. Acad. Sci. USA 2001, 98, 14268-14273. [PubMed: 11717396]

(23). Satoh A; Kim JK; Miyahara I; Devreese B; Vandenberghe I; Hacisalihoglu A; Okajima T; Kuroda S; Adachi O; Duine JA; Van Beeumen J; Tanizawa K; Hirotsu K Crystal Structure of Quinohemoprotein Amine Dehydrogenase from Pseudomonas Putida. Identification of a Novel Quinone Cofactor Encaged by Multiple Thioether Cross-Bridges. J. Biol. Chem. 2002, 277, 2830-2834. [PubMed: 11704672]

(24). Vandenberghe I; Kim JK; Devreese B; Hacisalihoglu A; Iwabuki H; Okajima T; Kuroda S; Adachi O; Jongejan JA; Duine JA; Tanizawa K; Van Beeumen J The Covalent Structure of the Small Subunit from Pseudomonas Putida Amine Dehydrogenase Reveals the Presence of Three Novel Types of Internal Cross-Linkages, All Involving Cysteine in a Thioether Bond. J. Biol. Chem 2001, 276, 42923-42931. [PubMed: 11555656]

(25). Nakai T; Ito H; Kobayashi K; Takahashi Y; Hori H; Tsubaki M; Tanizawa K; Okajima T The Radical S-Adenosyl-L-Methionine Enzyme QhpD Catalyzes Sequential Formation of IntraProtein Sulfur-to-Methylene Carbon Thioether Bonds. J. Biol. Chem 2015, 290, 11144-11166. [PubMed: 25778402]

(26). Caruso A; Bushin LB; Clark KA; Martinie RJ; Seyedsayamdost MR Radical Approach to Enzymatic $\beta$-Thioether Bond Formation. J. Am. Chem. Soc 2019, 141, 990-997. [PubMed: 30521328]

(27). Bruender NA; Wilcoxen J; Britt RD; Bandarian V Biochemical and Spectroscopic Characterization of a Radical S-Adenosyl-1-Methionine Enzyme Involved in the Formation of a Peptide Thioether Cross-Link. Biochemistry 2016, 55, 2122-2134. [PubMed: 27007615]

(28). Söding J; Biegert A; Lupas AN The HHpred Interactive Server for Protein Homology Detection and Structure Prediction. Nucleic Acids Res. 2005, 33, W244-W248. [PubMed: 15980461]

(29). Latham JA; Iavarone AT; Barr I; Juthani PV; Klinman JP PqqD Is a Novel Peptide Chaperone That Forms a Ternary Complex with the Radical S-Adenosylmethionine Protein PqqE in the Pyrroloquinoline Quinone Biosynthetic Pathway. J. Biol. Chem 2015, jbc.M115.646521.

(30). DiCaprio AJ; Firouzbakht A; Hudson GA; Mitchell DA Enzymatic Reconstitution and Biosynthetic Investigation of the Lasso Peptide Fusilassin. J. Am. Chem. Soc 2019, 141, 290297. [PubMed: 30589265]

(31). Hegemann JD; Schwalen CJ; Mitchell DA; Donk WA van der. Elucidation of the Roles of Conserved Residues in the Biosynthesis of the Lasso Peptide Paeninodin. Chem. Commun 2018, 54, 9007-9010.

(32). Mahanta N; Liu A; Dong S; Nair SK; Mitchell DA Enzymatic Reconstitution of Ribosomal Peptide Backbone Thioamidation. Proc. Natl. Acad. Sci 2018, 115, 3030-3035. [PubMed: 29507203] 
(33). Jang S; Imlay JA Hydrogen Peroxide Inactivates the Escherichia Coli Isc Iron-Sulphur Assembly System, and OxyR Induces the Suf System to Compensate. Mol. Microbiol 2010, 78, 1448-1467. [PubMed: 21143317]

(34). Hidalgo E; Bollinger JM; Bradley TM; Walsh CT; Demple B Binuclear [2Fe-2S] Clusters in the Escherichia Coli SoxR Protein and Role of the Metal Centers in Transcription. J. Biol. Chem 1995, 270, 20908-20914. [PubMed: 7673113]

(35). Brumby PE; Miller RW; Massey V The Content and Possible Catalytic Significance of Labile Sulfide in Some Metalloflavoproteins. J. Biol. Chem 1965, 240, 2222-2228. [PubMed: 14299651]

(36). Burkhart BJ; Kakkar N; Hudson GA; van der Donk WA; Mitchell DA Chimeric Leader Peptides for the Generation of Non-Natural Hybrid RiPP Products. ACS Cent. Sci 2017, 3, 629-638. [PubMed: 28691075] 


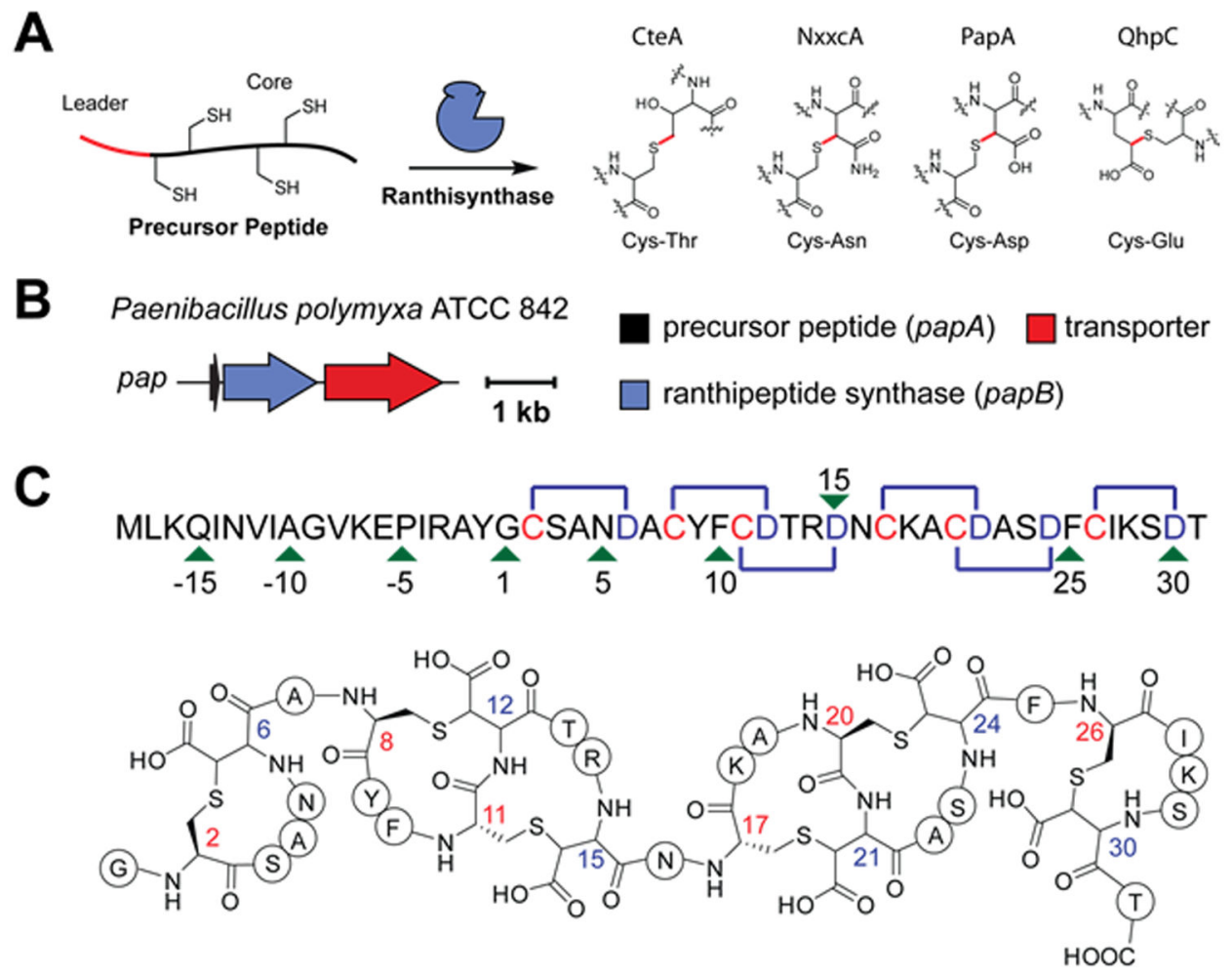

Figure 1. Ranthipeptide biosynthesis and freyrasin.

(A) Overview of ranthipeptide biosynthesis. Known thioether linkages (red) catalyzed by ranthipeptide rSAM enzymes are depicted. (B) Freyrasin BGC diagram. (C) Primary sequence of PapA with thioether linkages indicated by blue lines and expanded structure of freyrasin with Cys (red) and Asp (blue) colored. While all thioether linkages in freyrasin are known to be $S-C \beta$, the location of the leader peptide cleavage site (here shown between Tyr and Gly) is speculative. Negative numbering is used for the tentative leader region of PapA. CteA: thermocellin precursor; PapA: freyrasin precursor; QhpC: quinohemoprotein amine dehydrogenase small subunit precursor. 
A

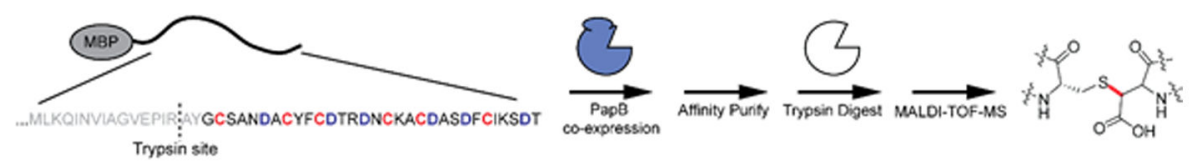

B
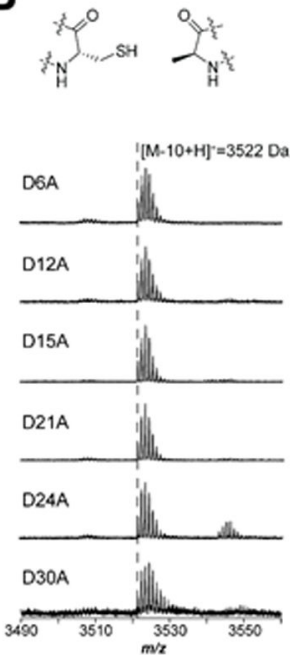

C

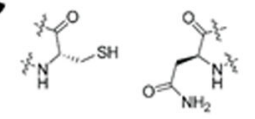

D

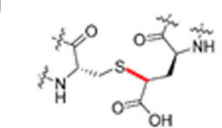

E
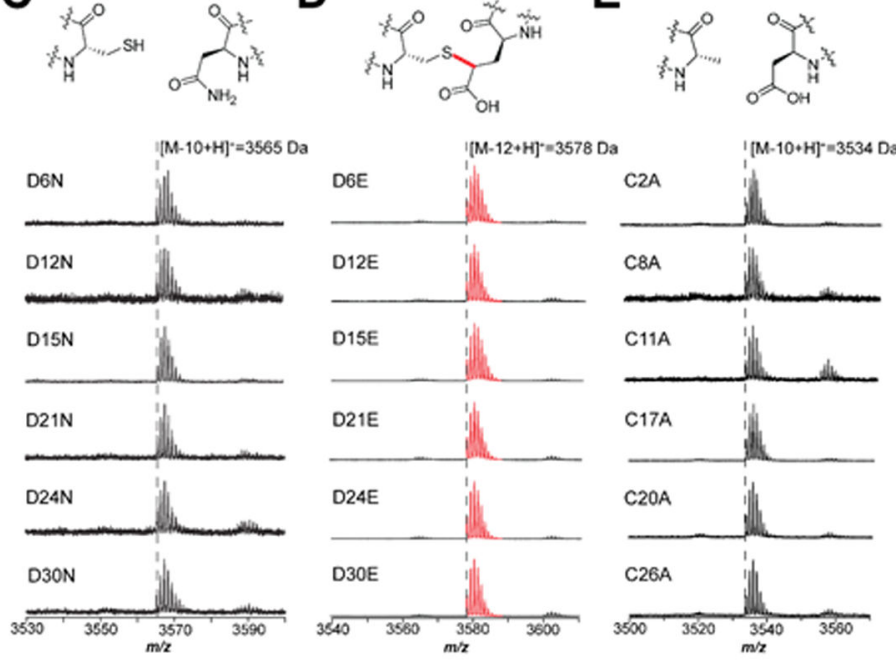

Figure 2. Determination of PapB substrate tolerance.

(A) Schematic overview for obtaining freyrasin variants. The primary sequence of WT PapA is shown for reference along with the WT thioether linkage installed by PapB. (B) MALDITOF-MS results of PapB reactions on PapA Asp to Ala scan with predicted substructures shown. (C) Same as (B), but with Asp to Asn variants. (D) Asp to Glu variants. (E) Cys to Ala variants. 
A

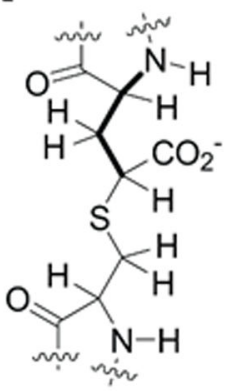

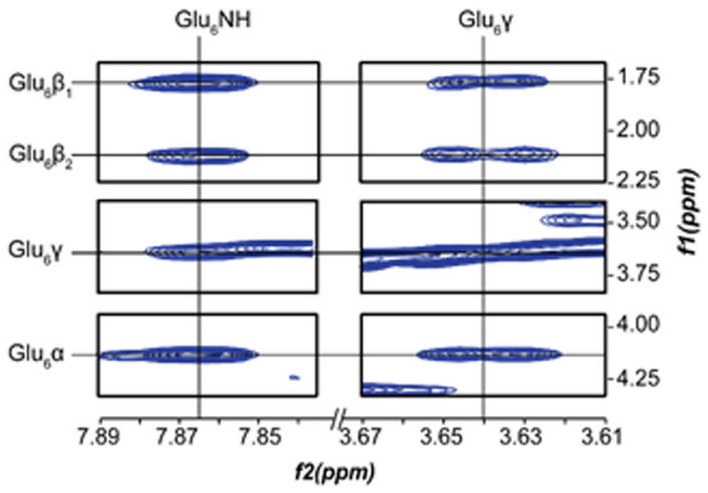

B

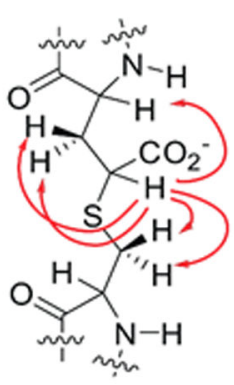

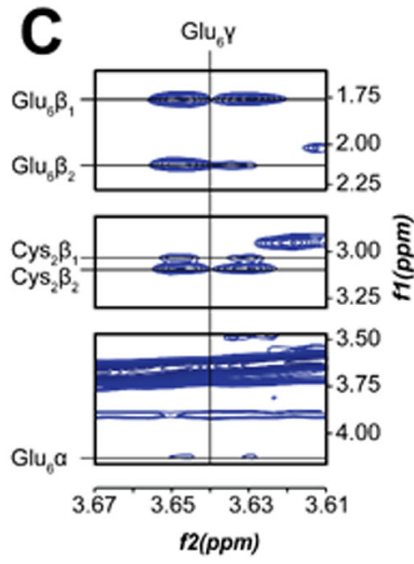

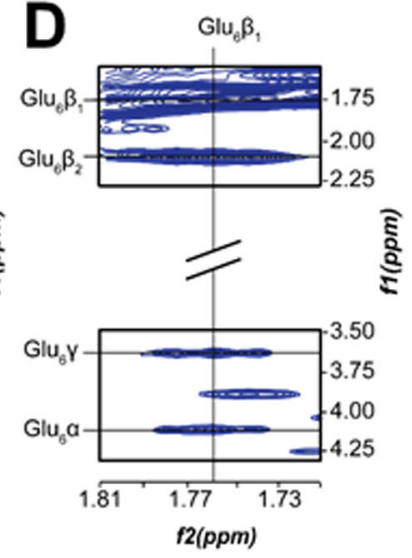

Figure 3. Multidimensional NMR analysis of PapA D6E variant.

(A) TOCSY of PapA-D6E modified by PapB. The chemical shifts in the spin system are most consistent with a $\gamma$-linkage. The Cys spin system is not shown here for clarity (see Figure S25) (B) Indicated NOE contacts for the crosslink-site proton in the ${ }^{1} \mathrm{H}-{ }^{1} \mathrm{H}$ NOESY. (C) NOE contacts visible for the crosslink $(\gamma)$ proton signal. All observed crosslinks support a thioether linkage at the $\gamma$ position on Glu6. (D) NOE contacts for Glu6 $\beta$ methylene protons. A strong NOE contact to both the $a$ and $\gamma$ positions supports its identity as a $\beta$ proton. 
A

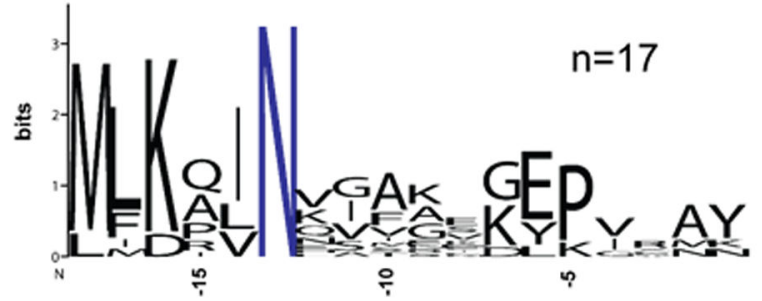

B

$\mathrm{MLKQINVIAGVKEPIRAY}_{-15}$
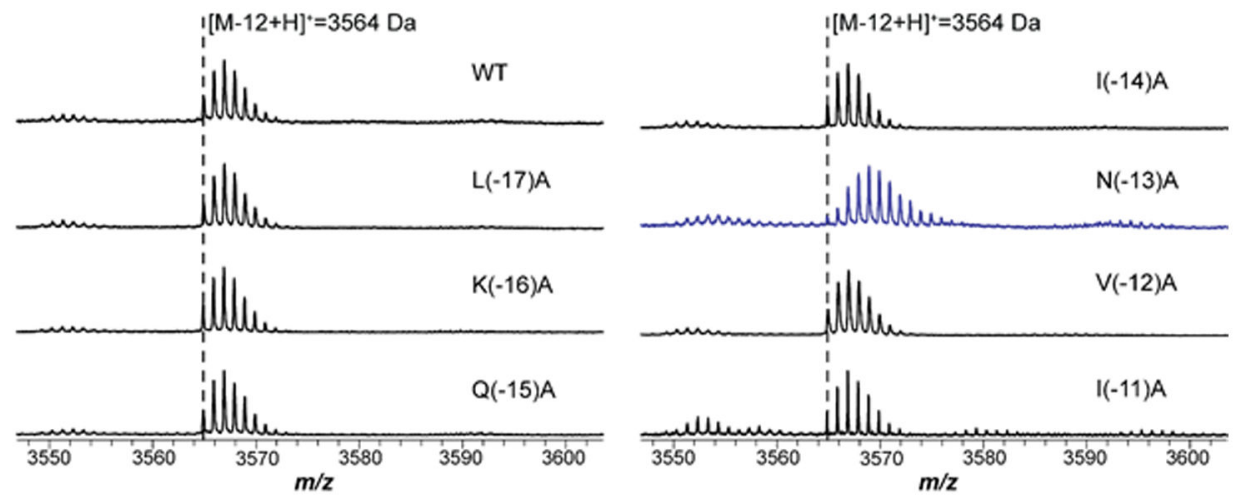

Figure 4. Thioether installation by PapB is leader-dependent.

(A) Leader peptide sequence logo generated for all 17 identified Cys- $\mathrm{X}_{3}$-Asp-like ranthipeptide precursors. ${ }^{7}$ (B) Mass of core peptide after co-expression with PapB as determined by MALDI-TOF-MS. Region of leader peptide subjected to Ala scan is indicated in red. These data support the hypothesis that PapB activity is hindered by the loss of $\operatorname{Asn}(-13)$ in vivo, but not abolished. 


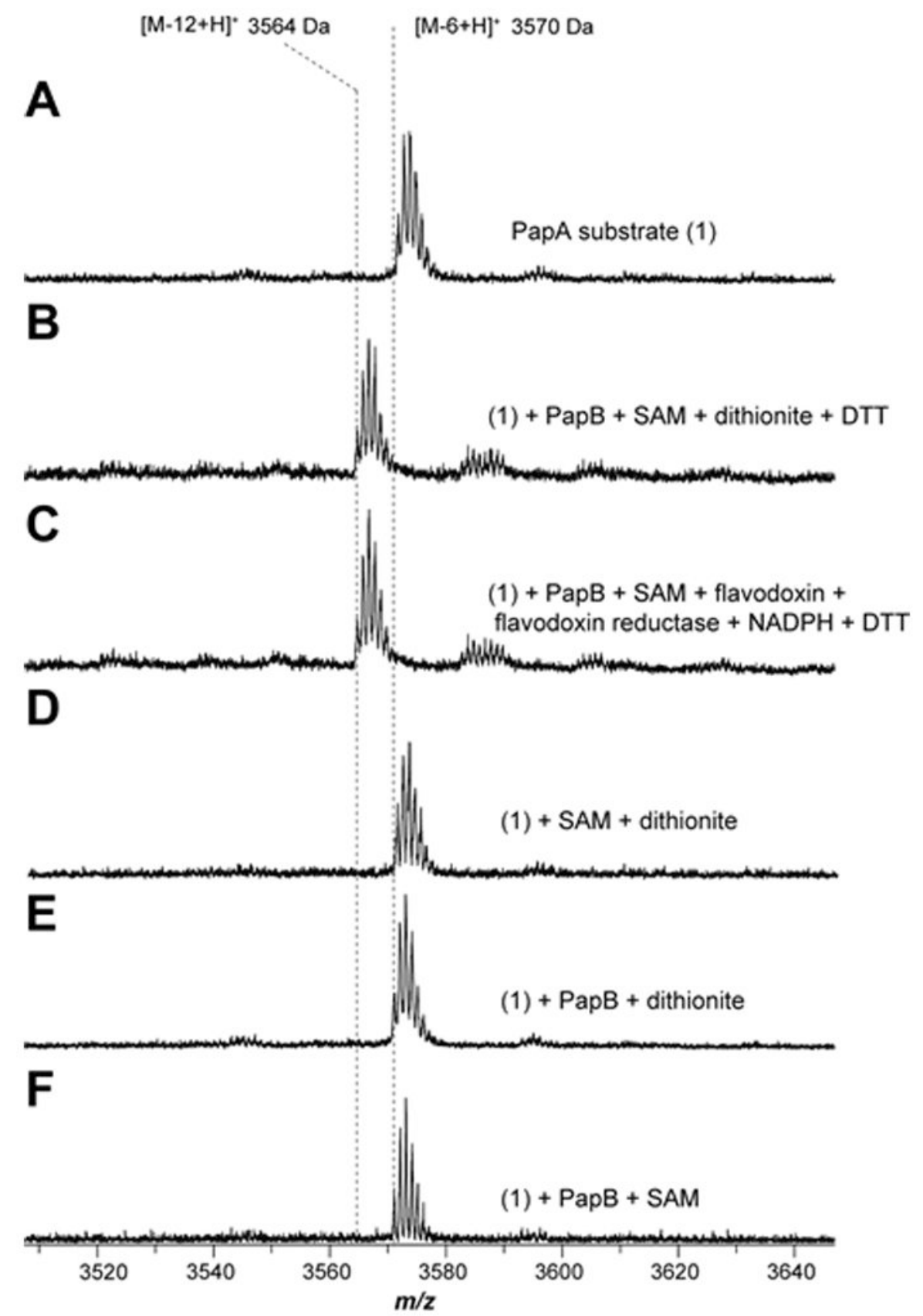

Figure 5: MALDI-TOF-MS analysis of PapA peptide treated with purified PapB in vitro. (A) Mass spectrum of the PapA peptide, $m / z 3570$ Da. Mass loss of $6 \mathrm{Da}$ is consistent with three disulfide bond formation as reported previously. ${ }^{7}$ (B) Mass spectrum of the peptide after reaction with the full array of reactants, $\mathrm{m} / \mathrm{z} 3564 \mathrm{Da}$. This is consistent with the formation of six thioether bonds (loss of $12 \mathrm{Da}$ from the unmodified peptide), similar to what is obtained from in vivo co-expression experiments. ${ }^{7}$ (C) Identical to B except flavodoxin, flavodoxin reductase and NADPH was used as the reductant. (D) No PapB control reaction. (E) No SAM control reaction. (F) No dithionite control reaction. 